\title{
Brain-derived neurotrophic factor and suicidal behavior in adolescents
}

\author{
Leo Sher \\ New York, USA
}

The p roblem of adolescent suicide

dolescent suicidal behavior is a medical and social problem worldwide. ${ }^{[1-4]}$ The tragedy of pediatric suicide is a national and global phenomenon. Each year about 100000 adolescents kill themselves worldwide (one every 5 minutes) in the context of 4 million suicide attempts.$^{[1,2]}$ According to the World Health Organization, it is among the top three causes of death in the population aged 15-34 years. ${ }^{[1]}$ In the USA, suicide is also a leading cause of death among those aged $15-24 .{ }^{[5]}$ Twenty percent to $25 \%$ of American adolescents have seriously considered suicide and $9 \%$ have tried it at least once. ${ }^{[6]}$ Suicide is rare in prepubertal children, but there is a precipitous rise in prevalence after puberty and through adolescence.

\section{Studies of adolescent suicide}

Although there has been an extensive research on the neurobiology of suicidal behavior in adults, few studies have focused on the neurobiological correlates of suicidal behavior in adolescents. Studies of adolescent suicide have mostly been focused on demographic and psychosocial risk factors. ${ }^{[7-10]}$ Such studies focus on who is at risk, but do not explain why certain adolescents are at risk for suicide. Studies of the neurobiology of adolescent suicide may explain why some adolescents are more suicidal than others and help to find biological markers of suicidal behavior in teenagers. Currently, we do not know the neurobiology of adolescent suicidality. We cannot treat and prevent suicidal behavior in

\footnotetext{
Author Affiliations: Department of Psychiatry, Mount Sinai School of Medicine and Department of Psychiatry, James J. Peters Veterans' Administration Medical Center, New York, USA (Sher L)

Corresponding Author: Leo Sher, MD, James J. Peters Veterans' Administration Medical Center, 130 West Kingsbridge Road, Bronx, New York 10468, USA (Tel: 718-584-9000x6821; Fax: 718-741-4703; Email: drleosher@gmail.com)

\section{doi:10.1007/s12519-011-0316-2}

CChildren's Hospital, Zhejiang University School of Medicine, China and Springer-Verlag Berlin Heidelberg 2011. All rights reserved.
}

adolescents if we do not know the psychobiology of youth suicidal behavior. Suicide prevention efforts should be confirmed by scientific knowledge.

\section{Brain-derived neurotrophic factor and adolescent suicidal behavior}

Over the past decade the role of brain-derived neurotrophic factor (BDNF) in the pathophysiology of suicidal behavior has attracted attention of scientists. ${ }^{[11-14]}$ Neurotrophic factors promote neuronal survival, regulate many aspects of neuronal development and function, including synapse formation and synaptic plasticity. ${ }^{[15]}$ Of various neurotrophic factors, BDNF has attracted a lot of interest because it is involved in the pathophysiology of many psychiatric disorders associated with suicidal behavior including depression, posttraumatic stress, schizophrenia, and obsessivecompulsive disorder. BDNF dysregulation may be associated with increased suicidality independently of psychiatric diagnoses. BDNF plays an important role in the regulation and growth of neurons during childhood and adolescence. ${ }^{[16]}$ Stressor-sensitive areas are undergoing developmental changes during adolescence. Acute and chronic stress inhibits hippocampal BDNF synthesis during brain development. ${ }^{[17]}$ The serotonin dysfunction found in adolescent and adult suicidal behavior may be related to the low level of BDNF, which impedes the normal development of serotonin neurons during brain development. A recent study demonstrated a down-regulation of both BDNF and its receptor TrkB in the prefrontal cortex and hippocampus of teenage suicide victims, which suggests that stress and altered BDNF may represent a major vulnerability factor in teenage suicidal behavior. ${ }^{[18]}$ Possibly, BDNF dysfunction plays a more significant part in the pathophysiology of psychiatric disorders and suicidal behavior in adolescents than in adults.

\section{Future directions}

Treatment-induced augmentation of BDNF function may reduce suicidal behavior secondary 
to the improvement in psychiatric disorders. It may also decrease suicidal behavior independently of improvement in psychiatric disorders. It is interesting to speculate that BDNF may be a biological marker of suicidal behavior in adolescents or in certain adolescent populations. Research on BDNF function in suicidal adolescents is one of the important study directions of the pathophysiology of suicidal and self-injurious behavior among young people. More neurobiological studies of psychiatric pathology in pediatric populations are needed.

Funding: None.

Ethical approval: Not needed.

Competing interest: None declared.

Contributors: Sher L is the sole author of the paper.

\section{References}

1 World Health Organization. The second decade: improving adolescent health and development. http://www.who.int/child adolescent_health/documents/frh_adh_98_18/en/index.html (accessed November 26, 2010).

2 Greydanus DE, Calles J Jr. Suicide in children and adolescents. Prim Care 2007;34:259-273.

3 Cash SJ, Bridge JA. Epidemiology of youth suicide and suicidal behavior. Curr Opin Pediatr 2009;21:613-619.

4 Sher L, Zalsman G. Alcohol and adolescent suicide. Int J Adolesc Med Health 2005; 17:197-203.

5 National Institute of Mental Health. Suicide in the U.S.: Statistics and Prevention. http://www.nimh.nih.gov/health/publications/ suicide-in-the-us-statistics-and-prevention/index.shtml (accessed November 2, 2010).

6 Zametkin AJ, Alter MR, Yemini T. Suicide in teenagers: assessment, management, and prevention. JAMA 2001;286:3120-3125.
7 Steele MM, Doey T. Suicidal behaviour in children and adolescents. Part 1: Etiology and risk factors. Can J Psychiatry 2007;52(6 Suppl 1):21S-33S.

8 Rutter PA, Behrendt AE. Adolescent suicide risk: four psychosocial factors. Adolescence 2004;39:295-302.

9 Brent DA, Baugher M, Bridge J. Age- and sex-related risk factors for adolescent suicide. J Am Acad Child Adolesc Psychiatry 1999;38:1497-1505.

10 Birmaher B, Ryan ND, Williamson DE, Brent DA, Kaufman J, Dahl RE, et al. Childhood and adolescent depression: a review of the past 10 years. Part I. J Am Acad Child Adolesc Psychiatry 1996;35:1427-1439.

11 Keller S, Sarchiapone M, Zarrilli F, Videtic A, Ferraro A, Carli $\mathrm{V}$, et al. Increased BDNF promoter methylation in the Wernicke area of suicide subjects. Arch Gen Psychiatry 2010;67:258-267.

12 Dawood T, Anderson J, Barton D, Lambert E, Esler M, Hotchkin E, et al. Reduced overflow of BDNF from the brain is linked with suicide risk in depressive illness. Mol Psychiatry 2007;12:981-983.

13 Dwivedi Y. Brain-derived neurotrophic factor: role in depression and suicide. Neuropsychiatr Dis Treat 2009;5:433-449.

14 Dwivedi Y. Brain-derived neurotrophic factor and suicide pathogenesis. Ann Med 2010;42:87-96.

15 Huang EJ, Reichardt LF. Neurotrophins: roles in neuronal development and function. Annu Rev Neurosci 2001;24:677736.

16 Mamounas LA, Blue ME, Siuciak JA, Altar CA. Brain-derived neurotrophic factor promotes the survival and sprouting of serotonergic axons in rat brain. J Neurosci 1995;15:7929-7939.

17 Murakami S, Imbe H, Morikawa Y, Kubo C, Senba E. Chronic stress, as well as acute stress, reduces BDNF mRNA expression in the rat hippocampus but less robustly. Neurosci Res 2005;53:129-139.

18 Pandey GN, Ren X, Rizavi HS, Conley RR, Roberts RC, Dwivedi Y. Brain-derived neurotrophic factor and tyrosine kinase $\mathrm{B}$ receptor signalling in post-mortem brain of teenage suicide victims. Int J Neuropsychopharmacol 2008;11:1047-1061.

Received February 6, 2011 Accepted after revision March 31, 2011 